\title{
A Mixed Finite Element Method for the Biharmonic Eigenvalue Problems of Plate Bending
}

\author{
By \\ Kazuo ISHIHARA*
}

\begin{abstract}
Summary
In this paper, we obtain error estimates of a finite element solution by means of the mixed method for the biharmonic eigenvalue problem of plate bending. Some numerical examples are also given.
\end{abstract}

\section{§1. Introduction}

Let us consider the finite element solution for the biharmonic eigenvalue problem of plate bending with the homogeneous Dirichlet boundary condition:

$$
\begin{array}{ll}
\Delta \Delta u=\lambda u & \text { in } \Omega, \\
u=\partial u / \partial n=0 & \text { on } \partial \Omega .
\end{array}
$$

Here $\Omega$ is a bounded convex domain in the two dimensional Euclidean space $\mathbf{R}^{2}$ with a smooth boundary $\partial \Omega, \Delta$ Laplacian and $\partial / \partial n$ differentiation in the outward normal direction to $\partial \Omega$.

In the finite element method for the static boundary value problem of plate bending:

$$
\begin{array}{ll}
\Delta \Delta w=f & \text { in } \Omega, \\
w=\partial w / \partial n=0 & \text { on } \partial \Omega,
\end{array}
$$

several schemes have been studied during the last few years. Here $f$ is a given function. Some of these schemes are the compatible model, the hybrid method, the mixed method, the non-conforming

* Department of Mathematics, Faculty of Science, Ehime University, Matsuyama 790, Japan. 
method and the discrete Kirchhoff assumption model (see [1], [2], [4], [6], [7], [9], [12]). However, neither the hybrid method nor the mixed method has been applied to the eigenvalue problem (1).

The purpose of this paper is to obtain error estimates for the finite element solutions of the eigenvalue problem (1), applying the mixed method with piecewise linear polynomials proposed by Miyoshi [9].

The plan of the present paper is as follows. In Section 2, we give some notations and facts about the eigenvalue problem for the biharmonic operator. In Section 3, we prove that the approximate eigenvalues and the corresponding eigenfunctions converge with a certain rate of convergence to the exact ones. Finally, in Section 4, some numerical results are also given to see the validity of our theory.

Throughout this paper, $C, C_{1}, C_{2}$ etc. are generic positive constants, independent of $h$, which are not necessarily the same at different places. Here $h$ is a parameter depending on a triangulation of the domain $\Omega$.

\section{§ 2. Preliminaries}

We assume that the solution $u$ of (1) is sufficiently smooth. Let $L_{2}(\Omega)$ be the real space of square integrable functions on $\Omega$. The inner product and the norm on $L_{2}(\Omega)$ are denoted by $($,$) and$ $\|\cdot\|$, respectively. Let $H^{n}(\Omega)$ be the real $n$-th order Sobolev space $(n=1,2, \cdots)$ with the norm given by

$$
\|u\|_{n}=\left(\sum_{|\alpha| \leq n}\left\|D^{a} u\right\|^{2}\right)^{\frac{1}{2}}
$$

where $\alpha=\left(\alpha_{1}, \alpha_{2}\right)$ is a multiple index with non-negative integers,

$$
|\alpha|=\alpha_{1}+\alpha_{2} \text { and } D^{\alpha}=\partial^{|\alpha|} / \partial x_{1}^{\alpha_{1}} \partial x_{2}^{\alpha_{2}} .
$$

We use the space $H_{0}^{n}(\Omega)$ which is the completion of the space of all test functions on $\Omega$ with respect to the norm of $H^{n}(\Omega)$.

The standard variational formulation of the eigenvalue problem (1) consists of finding a real eigenvalue $\lambda$ and a non-zero eigenfunction $u \in H_{0}^{2}(\Omega)$ such that 


$$
\langle u, \phi\rangle=\lambda(u, \phi) \text { for each } \phi \in H_{0}^{2}(\Omega)
$$

where

$$
\langle u, v\rangle=\sum_{|\alpha|=2}\left(D^{\alpha} u, D^{\alpha} v\right)
$$

It is well known that all the eigenvalues $\left\{\lambda_{i}\right\}$ of (3) are arranged as follows :

$$
0<\lambda_{1} \leqq \lambda_{2} \leqq \lambda_{3} \leqq \cdots<\infty
$$

The corresponding eigenfunctions are denoted by $\left\{u_{i}\right\}$ with the normalization condition

$$
\left(u_{i}, u_{j}\right)=\delta_{i j}
$$

where $\delta_{i j}$ is Kronecker's delta. It is also well known from the Rayleigh principle that the eigenvalues are characterized by

$$
\begin{aligned}
& \lambda_{1}=\min _{\substack{u \in H_{0}^{2}(\Omega) \\
u \neq 0}} \frac{\langle u, u\rangle}{(u, u)}=\min _{\substack{u \in H_{0}^{2}(\Omega) \\
u \neq 0}} \frac{(\Delta u, \Delta u)}{(u, u)}, \\
& \lambda_{i}=\min _{\substack{u \in H_{0}^{2}(\Omega) \\
u \neq 0 \\
(u, u j)=0 \\
j=1,2, \ldots, i-1}} \frac{\langle u, u\rangle}{(u, u)}, \quad i=2,3, \cdots \cdots .
\end{aligned}
$$

In order to introduce another variational formulation, we put (1) into the following problem:

$$
\begin{array}{ll}
v=\Delta u & \text { in } \Omega, \\
\Delta v=\lambda u & \text { in } \Omega, \\
u=\partial u / \partial n=0 & \text { on } \partial \Omega .
\end{array}
$$

The weak form of the problem (5) consists of finding a real eigenvalue $\lambda$ and non-zero eigenfunctions $u \in H_{0}^{1}(\Omega), v \in H^{1}(\Omega)$ such that

$$
\begin{aligned}
& a(u, \phi)+(v, \phi)=0 \text { for each } \phi \in H^{1}(\Omega), \\
& a(v, \phi)+\lambda(u, \phi)=0 \text { for each } \phi \in H_{0}^{1}(\Omega),
\end{aligned}
$$

where

$$
a(u, v)=\int_{\Omega}\left(\partial u / \partial x_{1} \cdot \partial v / \partial x_{1}+\partial u / \partial x_{2} \cdot \partial v / \partial x_{2}\right) d x_{1} d x_{2}
$$


Define the space $V$ as follows:

$$
\begin{aligned}
V= & \left\{\{u, v\} \in H_{0}^{1}(\Omega) \times H^{1}(\Omega) ; a(u, \phi)+(v, \phi)=0\right. \\
& \text { for each } \left.\phi \in H^{1}(\Omega)\right\} .
\end{aligned}
$$

Then, the eigenvalues $\lambda_{i}$ of (6) are characterized by

$$
\begin{aligned}
& \lambda_{1}=\min _{\substack{\{u, v\} \in V \\
u \neq 0}} \frac{\|v\|^{2}}{\|u\|^{2}},
\end{aligned}
$$

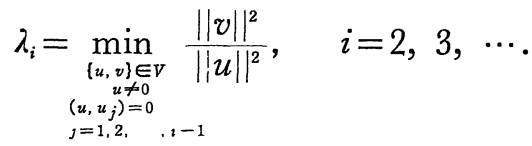

\section{§3. Finite Element Scheme and Rate of Convergence}

For simplicity, it is assumed that the domain $\Omega$ is a convex polygon. We decompose the domain $\Omega$ into $m$ disjoint triangular elements $\Delta_{k}(k=1,2, \cdots, m)$. By $P_{\imath}, 1 \leqq i \leqq n$, (or $\left.P_{\imath}, n+1 \leqq i \leqq n+J\right)$, we denote the nodal points of the triangulation $T^{h}$ which belong to $\Omega$ (or $\partial \Omega$ ). Here $h$ is the largest side length of all the triangular elements. We also assume that the triangulation $T^{h}$ satisfies the following conditions (see [9], [10]) :

(a) $\bar{\Omega}=\bigcup_{k=1}^{n} \bar{\Delta}_{k}, \Delta_{i} \cap \Delta_{j}=\varnothing(i \neq j)$.

(b) The minimum angle $\theta$ of all the triangles is bounded below in such a way that $\theta \geqq \theta_{0}>0$.

(c) There exists a closed subregion $\Omega_{h}=\cup_{k^{\prime}} \Delta_{k^{\prime}}$ of $\Omega$ which is composed of meshes of side length $\bar{h}$, and the number of the nodal points in $\Omega-\left(\Omega_{h}\right)_{\text {interior }}$ is of order $O\left(h^{-1}\right)$ as $h \rightarrow 0$. Each square in $\Omega_{h}$ is triangulated by the diagonal of north-east direction.

Let $\left\{\hat{\phi}_{i}\right\} \quad(i=1,2, \cdots, n+J)$ be the piecewise linear functions satisfying the relations

$$
\hat{\phi}_{i}\left(P_{j}\right)=\delta_{i j}, 1 \leqq i, j \leqq n+J
$$

Define finite dimensional spaces $Y^{h}$ and $Y_{0}^{h}$ as follows: 


$$
\begin{aligned}
& Y^{h}=\operatorname{Span}\left[\hat{\phi}_{1}, \hat{\phi}_{2}, \cdots, \hat{\phi}_{n+J}\right] \subset H^{1}(\Omega), \\
& Y_{0}^{h}=\left\{\hat{\phi} ; \hat{\phi} \in Y^{h}, \hat{\phi}=0 \text { on } \partial \Omega\right\} \subset H_{0}^{1}(\Omega) .
\end{aligned}
$$

For a given function $f \in L_{2}(\Omega)$, Miyoshi [9] proved that the mixed finite element solution $\left\{\hat{w}, \hat{w}_{1}\right\} \in Y_{0}^{h} \times Y^{h}$ defined by

$$
\begin{array}{ll}
a(\hat{w}, \hat{\phi})+\left(\hat{w}_{1}, \hat{\phi}\right)=0 & \text { for each } \hat{\phi} \in Y^{h}, \\
a\left(\hat{w}_{1}, \hat{\phi}\right)+(f, \hat{\phi})=0 & \text { for each } \hat{\phi} \in Y_{0}^{h},
\end{array}
$$

holds the following error estimate

$$
\|w-\hat{w}\|_{1}+\left\|w_{1}-\hat{w}_{1}\right\| \leqq C\|f\| h^{\frac{1}{2}},
$$

where $\left\{w, w_{1}=\Delta w\right\}$ is the exact solution of (2).

We define the finite element solution $\{\hat{\lambda}, \hat{u}, \hat{v}\}\left(\hat{u} \in Y_{0}^{h}, \hat{v} \in Y^{h}\right)$ of the consistent mass scheme for the eigenvalue problem (6) by

$$
\begin{array}{ll}
a(\hat{u}, \hat{\phi})+(\hat{v}, \hat{\phi})=0 & \text { for each } \hat{\phi} \in Y^{h}, \\
a(\hat{v}, \hat{\phi})+\hat{\lambda}(\hat{u}, \hat{\phi})=0 & \text { for each } \hat{\phi} \in Y_{0}^{h} .
\end{array}
$$

This scheme is equivalent to a set of the matrix eigenvalue equations

$$
\begin{aligned}
& K U+M V=0, \\
& K V+\hat{\lambda} M U=0 .
\end{aligned}
$$

Here $U=\left(\hat{u}\left(P_{i}\right)\right)$ and $V=\left(\hat{v}\left(P_{i}\right)\right)$ are unknown vectors, and $K$ and $M$ are the stiffness matrix and the consistent mass matrix given by

$$
\begin{array}{ll}
K=\left\{a\left(\hat{\phi}_{i}, \hat{\phi}_{j}\right)\right\}, & 1 \leqq i, j \leqq n+J, \\
M=\left\{\left(\hat{\phi}_{i}, \hat{\phi}_{j}\right)\right\}, & 1 \leqq i, j \leqq n+J .
\end{array}
$$

Let $V^{h}$ be a space defined by

$$
V^{h}=\left\{\{\hat{u}, \hat{v}\} \in Y_{0}^{h} \times Y^{h} ; a(\hat{u}, \hat{\phi})+(\hat{v}, \hat{\phi})=0 \text { for each } \hat{\phi} \in Y^{h}\right\} .
$$

Then the eigenvalues $\left\{\hat{\lambda}_{i}\right\}(i=1,2, \cdots, n)$ are characterized by

$$
\begin{aligned}
& \hat{\lambda}_{1}=\min _{\substack{\{a, b] \in v^{b} \\
u \neq 0}} \frac{\|\hat{\imath}\|^{2}}{\|\hat{u}\|^{2}},
\end{aligned}
$$

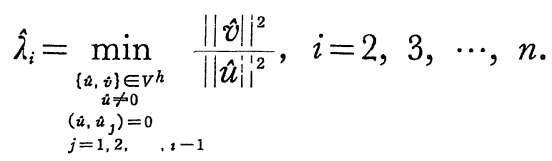

We can normalize the eigenfunctions $\hat{u}_{\imath}$ corresponding to $\hat{\lambda}_{\imath}$ by 


$$
\left(\hat{u}_{i}, \hat{u}_{j}\right)=\delta_{i j} \quad \text { and } \quad\left(u_{i}, \hat{u}_{i}\right) \geqq 0 \text {. }
$$

We shall prove the convergence of the approximate solutions for the first $n_{1}(\leqq n)$ eigenvalues and eigenfunctions. First we shall give error estimates for the approximate eigenvalues using the technique, analogous to the one used by Kikuchi $[6,7]$ and Strang and Fix [12]. Some lemmas are prepared.

\section{Lemma 1. Let}

$$
E_{i}=\left\{w_{i} ; w_{i} \in \operatorname{Span}\left[u_{1}, u_{2}, \cdots, u_{i}\right],\left\|w_{i}\right\|=1\right\}
$$

For $w_{i}=\sum_{j=1}^{i} \alpha_{j} u_{j} \in E_{\imath}$, define $\left\{\tilde{u}_{h i}, \tilde{v}_{h i}\right\} \in V^{h}$ by

$$
\begin{array}{ll}
a\left(\tilde{u}_{h i}, \hat{\phi}\right)+\left(\tilde{v}_{h i}, \hat{\phi}\right)=0 & \text { for each } \hat{\phi} \in Y^{h}, \\
a\left(\tilde{v}_{h i}, \hat{\phi}\right)+\left(\sum_{j=1}^{i} \alpha, \lambda, u_{j}, \hat{\phi}\right)=0 & \text { for each } \hat{\phi} \in Y_{0}^{h} .
\end{array}
$$

Then,

$$
\left\|\tilde{u}_{h i}-w_{i}\right\| \leqq C_{1} \lambda_{i} h^{\frac{1}{2}}
$$

Proof. By applying (8), the proof is complete.

Lemma 2. Let $S_{i}^{h}$ be an arbitrary $i$-dimensional subspace of $Y_{0}^{h}$. Let $u_{h}$ be an arbitrary non-zero element of $S_{i}^{h}(1 \leqq i \leqq n)$ such that

$$
\left(u_{h}, u_{j}\right)=0, j=1,2, \cdots, i-1 \text {. }
$$

When $i=1$, no constraint is imposed on $u_{h}$. Define $v_{h} \in Y^{h},\left\{u^{\prime}, v^{\prime}\right\} \in V$ and $\left\{u_{h}^{\prime}, v_{h}^{\prime}\right\} \in V^{h}$ by

$$
a\left(u_{h}, \hat{\phi}\right)+\left(v_{h}, \hat{\phi}\right)=0 \quad \text { for each } \hat{\phi} \in Y^{h},
$$

and

$$
\begin{array}{ll}
a\left(v^{\prime}, \phi\right)+\left(u_{h}, \phi\right)=0 & \text { for each } \phi \in H_{0}^{1}(\Omega), \\
a\left(u^{\prime}, \phi\right)+\left(v^{\prime}, \phi\right)=0 & \text { for each } \phi \in H^{1}(\Omega),
\end{array}
$$

and

$$
\begin{array}{ll}
a\left(v_{h}^{\prime}, \hat{\phi}\right)+\left(u_{h}, \hat{\phi}\right)=0 & \text { for each } \hat{\phi} \in Y_{0}^{h} \\
a\left(u_{h}^{\prime}, \hat{\phi}\right)+\left(v_{h}^{\prime}, \hat{\phi}\right)=0 & \text { for each } \hat{\phi} \in Y^{h}
\end{array}
$$


respectively. Then, $\left(u^{\prime}, u_{j}\right)=0, j=1,2, \cdots, i-1$, and

$$
\left\|\left.v_{h}^{\prime}\right|^{2} \leqq \lambda_{i}^{-1}\right\| u_{h} \|^{2}\left(1+C \lambda_{i} h^{\frac{1}{2}}\right) \text {. }
$$

Proof. From (8), we have

$$
\left\|u^{\prime}-u_{h}^{\prime}\right\| \leqq C\left\|u_{h}\right\| h^{\frac{1}{2}}
$$

By (17) and (16), we have

$$
\left\|v^{\prime}\right\|^{2}=-a\left(u^{\prime}, v^{\prime}\right)=\left(u_{h}, u^{\prime}\right) \leqq\left\|u_{h}\right\| \cdot\left\|u^{\prime}\right\| .
$$

Since $\left\{\lambda_{j}, u_{j}, v_{j}\right\}$ is the solution of (6), it holds that

$$
\begin{array}{lr}
a\left(u_{j}, \phi\right)+\left(v_{j}, \phi\right)=0 & \text { for each } \phi \in H^{1}(\Omega), \\
a\left(v_{j}, \phi\right)+\lambda_{,}\left(u_{,}, \phi\right)=0 & \text { for each } \phi \in H_{0}^{1}(\Omega) .
\end{array}
$$

Combining (22), (17), (21), (16) and (14), we obtain

$$
\begin{aligned}
\left(u_{j}, u^{\prime}\right) & =-\lambda_{j}^{-1} a\left(v_{j}, u^{\prime}\right)=\lambda_{j}^{-1}\left(v^{\prime}, v_{j}\right)=-\lambda_{j}^{-1} a\left(u_{j}, v^{\prime}\right) \\
& =\lambda_{j}^{-1}\left(u_{h}, u_{j}\right)=0, j=1,2, \cdots, i-1 .
\end{aligned}
$$

Therefore, from (7), we have

$$
\left.\lambda_{i}|| u^{\prime}\right|_{1} ^{2} \leqq\left\|v^{\prime}\right\|_{1}^{2} \leqq\left\|u_{h}\right\| \cdot\left\|u^{\prime}\right\| .
$$

Thus,

$$
\left\|u^{\prime}\right\| \leqq \lambda_{i}^{-1}\left\|u_{h}\right\|
$$

By (19) and (18), we obtain

$$
\begin{aligned}
\left\|v_{h}^{\prime}\right\|^{2} & =-a\left(u_{h}^{\prime}, v_{h}^{\prime}\right)=\left(u_{h}, u_{h}^{\prime}\right) \leqq\left\|u_{h}\right\| \cdot\left\|u_{h}^{\prime}\right\| \\
& \leqq\left\|u_{h}\right\|\left(\left\|u_{h}^{\prime}-u^{\prime}\right\|+\left\|u^{\prime}\right\|\right) \leqq\left\|u_{h}\right\|\left(C|| u_{h}\left\|h^{\frac{1}{2}}+\lambda_{\imath}^{-1}\right\| u_{h} \|\right) \\
& \leqq \lambda_{t}^{-1}|| u_{h} \|^{2}\left(1+C \lambda_{\imath} h^{\frac{1}{2}}\right) .
\end{aligned}
$$

This completes the proof.

We are now in a position to prove the following theorem.

Theorem 1. Let $\lambda_{i}$ and $\hat{\lambda}_{2}$ be the eigenvalues defined by (6) and (9), respectively. Then, for sufficiently small $h$,

$$
\left|\lambda_{i}-\hat{\lambda}_{i}\right| \leqq C_{2} \lambda_{i}^{2} h^{\frac{1}{2}}
$$

Proof. Define two mappings $Q: E_{\imath} \rightarrow Y^{h}$ and $R: E_{\imath} \rightarrow Y_{0}^{h}$ given 
by (12) and (11), respectively, that is,

$$
\tilde{v}_{h i}=Q w_{i}, \quad \tilde{u}_{h i}=R w_{i} .
$$

By (11), (12) and (13), we have

$$
\begin{aligned}
\left\|\tilde{v}_{h i}\right\|^{2} & =-a\left(\tilde{u}_{h i}, \tilde{v}_{h i}\right)=\left(\sum_{j=1}^{i} \alpha_{j} \lambda_{j} u_{j}, \tilde{u}_{h i}\right) \leqq \lambda_{i}\left\|\sum_{j=1}^{i} \alpha_{j} u_{j} ! \cdot\right\| \tilde{u}_{h i} \| \\
& =\lambda_{i}|| w_{i}\|\cdot\| \tilde{u}_{h i}\left\|\leqq \lambda_{i}|| \tilde{u}_{h i}\right\|\left(i\left|w_{i}-\tilde{u}_{h i}\|+\| \tilde{u}_{h i}\right|\right) \\
& \leqq \lambda_{i}\left\|\tilde{u}_{h i}\right\|^{2}\left(1+C \lambda_{i} h^{\frac{1}{2}}\right) .
\end{aligned}
$$

Thus,

$$
\frac{\left\|Q w_{i}\right\|^{2}}{\left\|R w_{i}\right\|^{2}}=\frac{\left\|\tilde{v}_{h i}\right\|^{2}}{\left\|\tilde{u}_{h i}\right\|^{2}} \leqq \lambda_{i}\left(1+C \lambda_{i} h^{\frac{1}{2}}\right)
$$

Since $S_{i}=R E_{i}$ is $i$-dimensional, an application of the min-max principle $([12])$ gives

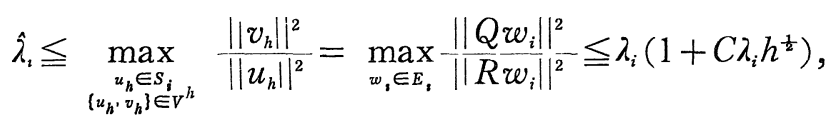

for sufficiently small $h$.

On the other hand, from (18) and (15), we have

$$
\left\|u_{h}\right\|^{2}=-a\left(v_{h}^{\prime}, u_{h}\right)=\left(v_{h}, v_{h}^{\prime}\right) \leqq\left\|v_{h}\right\| \cdot\left\|v_{h}^{\prime}\right\| \cdot
$$

Thus, using Lemma 2 yields

$$
\left\|u_{h}\right\|^{4} \leqq\left.\left\|v_{h}\right\|^{2}|| v_{h}^{\prime}\right|^{2} \leqq\left\|v_{h}\right\|^{2} \lambda_{i}^{-1}|| u_{h} \|^{2}\left(1+C \lambda_{i} h^{\frac{1}{2}}\right) .
$$

Therefore, we obtain

$$
\frac{\left\|v_{h}\right\|^{2}}{\left\|u_{h}\right\|^{2}} \geqq \lambda_{i}\left(1+C \lambda_{i} h^{\frac{1}{2}}\right)^{-1} \geqq \lambda_{i}\left(1-C \lambda_{i} h^{\frac{1}{2}}\right) .
$$

Thus, for sufficiently small $h$, using the min-max principle gives

$$
\hat{\lambda}_{i} \geqq \lambda_{i}\left(1-C \lambda_{i} h^{\frac{1}{2}}\right) \text {. }
$$

By combining (23) and (24), the proof is complete.

We next give error estimates for the approximate eigenfunctions. For $u_{\imath}$, define $\left\{\hat{z}_{i}, \hat{w}_{h i}\right\} \in V^{h}$ by

$$
\begin{array}{ll}
a\left(\hat{z}_{i}, \hat{\phi}\right)+\left(\hat{w}_{h i}, \hat{\phi}\right)=0 & \text { for each } \hat{\phi} \in Y^{h} \\
a\left(\hat{w}_{h i}, \hat{\phi}\right)+\lambda_{\imath}\left(u_{i}, \hat{\phi}\right)=0 & \text { for each } \hat{\phi} \in Y_{0}^{h}
\end{array}
$$


Then, from Lemma 1, we have

$$
\left\|\hat{z}_{i}-u_{i}\right\| \leqq C_{1} \lambda_{i} h^{\frac{1}{2}}=C_{3} h^{\frac{1}{2}} .
$$

Making use of the fact that $\left\{\hat{u}_{1}, \hat{u}_{2}, \cdots, \hat{u}_{n}\right\}$ are orthonormal in the norm ii $\|$, we expand

$$
\hat{z}_{i}=\sum_{k=1}^{i-1}\left(\hat{z}_{i}, \hat{u}_{k}\right) \hat{u}_{k}+\left(\hat{z}_{i}, \hat{u}_{i}\right) \hat{u}_{i}+\hat{f}_{i}^{\prime \prime} .
$$

For $\hat{f}_{i}^{\prime \prime} \in Y_{0}^{h}$, define $\hat{g}_{i}^{\prime \prime} \in Y^{h}$ by

$$
a\left(\hat{f}_{i}^{\prime \prime}, \hat{\phi}\right)+\left(\hat{g}_{i}^{\prime \prime}, \hat{\phi}\right)=0 \text { for each } \hat{\phi} \in Y^{h} .
$$

Then, we obtain the following two lemmas for the estimates of $\left\|\hat{f}_{i}^{\prime \prime}\right\|$ and $\left\|\sum_{k=1}^{i-1}\left(\hat{z}_{i}, \hat{u}_{k}\right) \hat{u}_{k}\right\|$.

Lemma 3. Suppose that $\lambda_{i}<\lambda_{i+1}$. Write $\hat{z}_{i}$ as (28). Then, for sufficiently small $h$,

$$
\left\|\hat{f}_{i}^{\prime \prime}\right\| \leqq C_{4} h^{\frac{1}{2}}
$$

Proof. Consider the quantity $\left\|\hat{g}_{i}^{\prime \prime}\right\|^{2}-\lambda_{i}|| \hat{f}_{i}^{\prime \prime} \|^{2}$. By (28), we have

$$
\left(\hat{f}_{i}^{\prime \prime}, \hat{u}_{k}\right)=0, \quad 1 \leqq k \leqq i \text {. }
$$

Since $\left\{\hat{\lambda}_{k}, \hat{u}_{k}, \hat{v}_{k}\right\}$ are the solutions of (9), it holds that

$$
\begin{array}{ll}
a\left(\hat{u}_{k}, \hat{\phi}\right)+\left(\hat{v}_{k}, \hat{\phi}\right)=0 & \text { for each } \hat{\phi} \in Y^{h}, \\
a\left(\hat{v}_{k}, \hat{\phi}\right)+\hat{\lambda}_{k}\left(\hat{u}_{k}, \hat{\phi}\right)=0 & \text { for each } \hat{\phi} \in Y_{0}^{h} .
\end{array}
$$

By (29), (32) and (30), we have

$$
\left(\hat{g}_{i}^{\prime \prime}, \hat{v}_{k}\right)=-a\left(\hat{f}_{i}^{\prime \prime}, \hat{v}_{k}\right)=\hat{\lambda}_{k}\left(\hat{u}_{k}, \hat{f}_{i}^{\prime \prime}\right)=0, \quad 1 \leqq k \leqq i .
$$

From (28) and (30), we have

$$
\left(\hat{z}_{i}, \hat{f}_{i}^{\prime \prime}\right)=\sum_{k=1}^{i}\left(\hat{z}_{i}, \hat{u}_{k}\right)\left(\hat{u}_{k}, \hat{f}_{i}^{\prime \prime}\right)+\left\|\hat{f}_{i}^{\prime \prime}\right\|^{2}=\left\|\hat{f}_{i}^{\prime \prime}\right\|^{2} .
$$

Combining (25), (28), (31), (29) and (33), we have

$$
\begin{aligned}
\left(\hat{w}_{h i}, \hat{g}_{i}^{\prime \prime}\right) & =-a\left(\hat{z}_{i}, \hat{g}_{i}^{\prime \prime}\right)=-\sum_{k=1}^{i}\left(\hat{z}_{i}, \hat{u}_{k}\right) \cdot a\left(\hat{u}_{k}, \hat{g}_{i}^{\prime \prime}\right)-a\left(\hat{f}_{i}^{\prime \prime}, \hat{g}_{i}^{\prime \prime}\right) \\
& =\sum_{k=1}^{i}\left(\hat{z}_{i}, \hat{u}_{k}\right)\left(\hat{v}_{k}, \hat{g}_{i}^{\prime \prime}\right)+\left\|\hat{g}_{i}^{\prime \prime}\right\|^{2}=\left\|\hat{g}_{i}^{\prime \prime}\right\|^{2} .
\end{aligned}
$$

Therefore, from (34), (35), (29), (26), we obtain 


$$
\begin{aligned}
\left\|\hat{g}_{i}^{\prime \prime}\right\|^{2} & -\lambda_{i}\left\|\hat{f}_{i}^{\prime \prime}\right\|^{2}=\left(\hat{w}_{h i}, \hat{g}_{i}^{\prime \prime}\right)-\lambda_{i}\left(\hat{z}_{i}, \hat{f}_{i}^{\prime \prime}\right) \\
& =-a\left(\hat{f}_{i}^{\prime \prime}, \hat{w}_{h \imath}\right)-\lambda_{i}\left(\hat{z}_{i}, \hat{f}_{i}^{\prime \prime}\right)=\lambda_{i}\left(u_{i}, \hat{f}_{i}^{\prime \prime}\right)-\lambda_{i}\left(\hat{z}_{i}, \hat{f}_{i}^{\prime \prime}\right) \\
& =\lambda_{i}\left(u_{2}-\hat{z}_{i}, \hat{f}_{i}^{\prime \prime}\right) \leqq \lambda_{i}\left\|u_{i}-\hat{z}_{i}\right\| \cdot\left\|\hat{f}_{i}^{\prime \prime}\right\| .
\end{aligned}
$$

Combining (10), (29) and (30) gives

$$
|| \hat{g}_{i}^{\prime \prime}\left\|^{2} \geqq \hat{\lambda}_{i+1}|| \hat{f}_{i}^{\prime \prime}\right\|^{2}
$$

Thus, from (36) and (37), we have

$$
\left(\hat{\lambda}_{i+1}-\lambda_{i}\right)\left\|\hat{f}_{i}^{\prime \prime}\right\| \leqq \lambda_{i}\left\|u_{i}-\hat{z}_{i}\right\| \text {. }
$$

On the other hand, by Theorem 1 and $\lambda_{i+1}>\lambda_{i}$, we obtain

$$
\hat{\lambda}_{i+1}-\lambda_{i}>\left(\lambda_{i+1}-\lambda_{i}\right) / 2>0
$$

for sufficiently small $h$. Therefore, applying (27) yields

$$
\left\|\hat{f}_{i}^{\prime \prime}\right\| \leqq \frac{2 \lambda_{i}}{\lambda_{i+1}-\lambda_{i}}\left\|u_{i}-\hat{z}_{i}\right\| \leqq C_{4} h^{\frac{1}{2}}
$$

for sufficiently small $h$. This completes the proof.

We now obtain an estimate of $\left\|\sum_{k=1}^{i-1}\left(\hat{z}_{i}, \hat{u}_{k}\right) \hat{u}_{k}\right\|_{i}$

Lemma 4. Suppose $\lambda_{i}>\lambda_{r_{-1}}$. Write $\hat{z}_{i}$ as (28). Define $\left\{\hat{f}_{i}^{\prime}, \hat{g}_{i}^{\prime}\right\}$ $\in V^{h}$ by

$$
\begin{gathered}
\hat{f}_{i}^{\prime}=\sum_{k=1}^{i-1}\left(\hat{z}_{i}, \hat{u}_{k}\right) \hat{u}_{k}, \\
a\left(\hat{f}_{i}^{\prime}, \hat{\phi}\right)+\left(\hat{g}_{i}^{\prime}, \hat{\phi}\right)=0 \quad \text { for each } \hat{\phi} \in Y^{h} .
\end{gathered}
$$

Then, for sufficiently small $h$,

$$
\left\|\hat{f}_{i}^{\prime}\right\| \leqq C_{5} h^{\frac{1}{2}}
$$

Proof. Consider the quantity $-\left\|\hat{g}_{i}^{\prime}\right\|^{2}+\lambda_{i} \mid \hat{f}_{i}^{\prime} \|^{2}$. From (39), (38), (31), (25) and (32), we have

$$
\begin{aligned}
\left(\hat{w}_{h i}, \hat{g}_{i}^{\prime}\right) & =-a\left(\hat{f}_{i}^{\prime}, \hat{w}_{h i}\right)=-\sum_{k=1}^{i-1}\left(\hat{z}_{i}, \hat{u}_{k}\right) a\left(\hat{u}_{k}, \hat{w}_{h i}\right)=\sum_{k=1}^{i-1}\left(\hat{z}_{i}, \hat{u}_{k}\right)\left(\hat{v}_{k}, \hat{w}_{h i}\right) \\
& =-\sum_{k=1}^{i-1}\left(\hat{z}_{i}, \hat{u}_{k}\right) a\left(\hat{z}_{i}, \hat{v}_{k}\right)=\sum_{k=1}^{i-1}\left(\hat{z}_{i}, \hat{u}_{k}\right) \hat{\lambda}_{k}\left(\hat{u}_{k}, \hat{z}_{i}\right) \\
& =\sum_{k=1}^{i-1} \hat{\lambda}_{k}\left(\hat{z}_{i}, \hat{u}_{k}\right)^{2} .
\end{aligned}
$$


From (39), (38), (31), (39), (38) and (32) we have

$$
\begin{aligned}
\left\|\hat{g}_{i}^{\prime}\right\|^{2} & =-a\left(\hat{f}_{i}^{\prime}, \hat{g}_{i}^{\prime}\right)=-\sum_{k=1}^{i-1}\left(\hat{z}_{i}, \hat{u}_{k}\right) a\left(\hat{u}_{k}, \hat{g}_{i}^{\prime}\right)=\sum_{k=1}^{i-1}\left(\hat{z}_{i}, \hat{u}_{k}\right)\left(\hat{v}_{k}, \hat{g}_{i}^{\prime}\right) \\
& =-\sum_{k=1}^{i-1}\left(\hat{z}_{i}, \hat{u}_{k}\right) a\left(\hat{f}_{i}^{\prime}, \hat{v}_{k}\right)=-\sum_{k=1}^{i-1}\left(\hat{z}_{i}, \hat{u}_{k}\right) a\left(\sum_{j=1}^{i-1}\left(\hat{z}_{i}, \hat{u}_{j}\right) \hat{u}_{j}, \hat{v}_{k}\right) \\
& =-\sum_{k, j=1}^{i-1}\left(\hat{z}_{i}, \hat{u}_{k}\right)\left(\hat{z}_{i}, \hat{u}_{j}\right) a\left(\hat{u}_{j}, \hat{v}_{k}\right) \\
& =\sum_{k, j=1}^{i-1}\left(\hat{z}_{i}, \hat{u}_{k}\right)\left(\hat{z}_{i}, \hat{u}_{j}\right) \hat{\lambda}_{k}\left(\hat{u}_{k}, \hat{u}_{j}\right)=\sum_{k=1}^{i-1} \hat{\lambda}_{k}\left(\hat{z}_{i}, \hat{u}_{k}\right)^{2} .
\end{aligned}
$$

Thus,

$$
\left\|\hat{g}_{i}^{\prime}\right\|^{2}=\left(\hat{w}_{h i}, \hat{g}_{i}^{\prime}\right) \text {. }
$$

By (38), we have

$$
\left(\hat{z}_{i}, \hat{f}_{i}^{\prime}\right)=\sum_{k=1}^{i-1}\left(\hat{z}_{i}, \hat{u}_{k}\right)^{2}=\left\|\hat{f}_{i}^{\prime}\right\|^{2}
$$

Combining (40), (41), (39), (26), we obtain

$$
\begin{aligned}
-\left\|\hat{g}_{i}^{\prime}\right\|^{2} & +\lambda_{i}\left\|\hat{f}_{i}^{\prime}\right\|^{2}=-\left(\hat{w}_{h i}, \hat{g}_{i}^{\prime}\right)+\lambda_{i}\left(\hat{z}_{i}, \hat{f}_{i}^{\prime}\right) \\
& =a\left(\hat{w}_{h i}, \hat{f}_{i}^{\prime}\right)+\lambda_{i}\left(\hat{z}_{i}, \hat{f}_{i}^{\prime}\right)=-\lambda_{i}\left(u_{i}, \hat{f}_{i}^{\prime}\right)+\lambda_{i}\left(\hat{z}_{i}, \hat{f}_{i}^{\prime}\right) \\
& =\lambda_{i}\left(\hat{z}_{i}-u_{i}, \hat{f}_{i}^{\prime}\right) \leqq \lambda_{i}\left\|u_{i}-\hat{z}_{i}\right\| \cdot\left\|\hat{f}_{i}^{\prime}\right\| .
\end{aligned}
$$

By the property of the eigenvalue and (38) and (39), we have

$$
\left\|\hat{g}_{i}^{\prime}\right\|^{2} \leqq \hat{\lambda}_{i-1}\left\|\hat{f}_{i}^{\prime}\right\|^{2}
$$

Thus, from (42) and (43), we obtain

$$
\left(\lambda_{i}-\hat{\lambda}_{i-1}\right)\left\|\hat{f}_{i}^{\prime}\right\| \leqq \lambda_{i}\left\|u_{i}-\hat{z}_{i}\right\| \text {. }
$$

On the other hand, by Theorem 1 and $\lambda_{i}>\lambda_{i-1}$, for sufficiently small $h$, we have

$$
\lambda_{i}-\hat{\lambda}_{i-1}>\left(\lambda_{i}-\lambda_{i-1}\right) / 2>0 .
$$

Therefore, applying (27) yields

$$
\left\|\hat{f}_{i}^{\prime}\right\| \leqq-\frac{2 \lambda_{i}}{\lambda_{i}-\lambda_{i-1}}\left\|u_{i}-\hat{z}_{i}\right\| \leqq C_{5} h^{\frac{1}{2}} .
$$

for sufficiently small $h$. The proof is complete.

We now prove the following theorem. 
Theorem 2. Let $\lambda_{i}$ be an eigenvalue of multiplicity $p+1(p \geqq 0$, $\left.\lambda_{i-1}<\lambda_{i}=\lambda_{i+1}=\cdots=\lambda_{i+p}<\lambda_{i+p+1}\right)$ of (6) and $u_{i}, u_{i+1}, \cdots, u_{i+p}$ be the corresponding eigenfunctions. Let $\hat{u}_{k}$ be the approximate eigenfunction corresponding to $\hat{\lambda}_{k}(k=1,2, \cdots, n)$. Then, for sufficiently small $h$,

$$
\operatorname{dist}\left\{u_{j}, \operatorname{Span}\left[\hat{u}_{i}, \hat{u}_{i+1}, \cdots, \hat{u}_{i+p}\right]\right\} \leqq C_{6} h^{\frac{1}{2}},
$$

$j=i, i+1, \cdots, i+p$, where

$$
\operatorname{dist}\{u, B\}=\inf _{u \in B}\|u-\hat{u}\| \text {. }
$$

Proof. Define $\hat{u}_{j}^{*} \in \operatorname{Span}\left[\hat{u}_{i}, \cdots, \hat{u}_{i+p}\right]$ by

$$
\hat{u}_{j}^{*}=\sum_{k=i}^{i+p}\left(u_{j}, \hat{u}_{k}\right) \hat{u}_{k}, \quad j=i, i+1, \cdots, i+p .
$$

For $u$, define $\left\{\hat{z}_{j}, \hat{w}_{h}\right\} \in V^{h}$ by (25) and (26) $(j=i, i+1, \cdots, i+p)$. Then, from (27)

$$
\left\|u_{j}-\hat{z}_{j}\right\| \leqq C_{3} h^{\frac{1}{2}}, \quad j=i, i+1, \cdots, i+p .
$$

Write $\hat{z}_{j}$ in the form

$$
\hat{z}_{j}=\sum_{k=1}^{i-1}\left(\hat{z}_{j}, \hat{u}_{k}\right) \hat{u}_{k}+\sum_{k=1}^{i+p}\left(\hat{z}_{j}, \hat{u}_{k}\right) \hat{u}_{k}+\hat{f}_{j}
$$

Since $\lambda_{i+p}<\lambda_{i+p+1}$ and since $u_{j}$ is the eigenfunction corresponding to $\lambda_{i+p}$, an application of Lemma 3 gives

$$
\| \hat{f}_{j} \mid \leqq C_{4} h^{\frac{1}{2}}
$$

for sufficiently small $h$. Similarly, since $\lambda_{i-1}<\lambda_{i}$ and $u_{j}$ is the eigenfunction corresponding to $\lambda_{i}$, an application of Lemma 4 gives

$$
\left\|\sum_{k=1}^{i-1}\left(\hat{z}_{j}, \hat{u}_{k}\right) \hat{u}_{k}\right\| \leqq C_{5} h^{\frac{1}{2}}
$$

for sufficiently small $h$. Therefore we have

$$
\begin{aligned}
\left\|\hat{u}_{j}^{*}-\hat{z}_{j}\right\| & \leqq\left\|\sum_{k=1}^{i-1}\left(\hat{z}_{j}, \hat{u}_{k}\right) \hat{u}_{k}\right\|+\left\|\sum_{k=i}^{i+p}\left(u_{j}-\hat{z}_{j}, \hat{u}_{k}\right) \hat{u}_{k}\right\|+\left\|\hat{f}_{j}\right\| \\
& \leqq C_{5} h^{\frac{1}{2}}+(p+1) \| u,-\hat{z}_{j}||+C_{4} h^{\frac{1}{2}} \\
& \leqq C_{5} h^{\frac{1}{2}}+(p+1) C_{3} h^{\frac{1}{2}}+C_{4} h^{\frac{1}{2}}=C h^{\frac{1}{2}}
\end{aligned}
$$

Thus, by the triangle inequality we obtain

$$
\left\|u_{j}-\hat{u}_{j}^{*}\right\| \leqq\left\|u_{j}-\hat{z}_{j}\right\|+\left\|\hat{z}_{j}-\hat{u}_{j}^{*}\right\| \leqq C_{6} h^{\frac{1}{2}}, \quad j=i, i+1, \cdots, i+p,
$$


for sufficiently small $h$. The proof is complete.

We can now prove the following corollary to Theorem 2.

Corollary. If $\lambda_{i}$ is a simple eigenvalue, then, for sufficiently small $h$,

$$
\left\|u_{i}-\hat{u}_{i}\right\| \leqq C_{7} h^{\frac{1}{2}}
$$

Proof. Putting $\beta_{i}=\left(u_{i}, \hat{u}_{i}\right) \geqq 0$, define $\hat{u}_{i}^{*}$ by

$$
\hat{u}_{i}^{*}=\beta_{i} \hat{u}_{i} .
$$

Then, from the proof of Theorem 2, we have

$$
\left\|u_{i}-\hat{u}_{i}^{*}\right\| \leqq C_{6} h^{\frac{1}{2}}
$$

for sufficiently small $h$. Moreover, we have

$$
\begin{aligned}
\left\|u_{i}-\hat{u}_{i}^{*}\right\|^{2} & =\left\|u_{i}\right\|^{2}-2\left(u_{i}, \hat{u}_{i}^{*}\right)+\left\|\hat{u}_{i}^{*}\right\|^{2}=1-2 \beta_{i}^{2}+\beta_{i}^{2} \\
& =1-\beta_{i}^{2} .
\end{aligned}
$$

Using the triangle inequality, for sufficiently small $h$, we obtain

$$
\begin{aligned}
& \left\|u_{\imath}-\hat{u}_{i}\right\| \leqq\left\|u_{\imath}-\beta_{\imath} \hat{u}_{i}\right\|+\left\|\beta_{\imath} \hat{u}_{i}-\hat{u}_{\imath}\right\| \\
& \quad=\left\|u_{\imath}-\hat{u}_{i}^{*}\right\|+1-\beta_{i}=\left\|u_{\imath}-\hat{u}_{\imath}^{*}\right\|+\frac{\left\|u_{i}-\hat{u}_{i}^{*}\right\|^{2}}{1+\beta_{i}} \\
& \quad \leqq\left\|u_{\imath}-\hat{u}_{\imath}^{*} \mid+\right\| u_{\imath}-\hat{u}_{\imath}^{*} \|^{2} \leqq C_{7} h^{\frac{1}{2}} .
\end{aligned}
$$

This completes the proof.

Remark. Further, we can propose the generalized mixed mass scheme with a parameter $\beta, 0 \leqq \beta \leqq 1$ (see [15]) :

$$
\begin{aligned}
& K U+\left\{\beta M+(1-\beta) M_{1}\right\} V=0, \\
& K V+\tilde{\lambda}\left\{\beta M+(1-\beta) M_{1}\right\} U=0,
\end{aligned}
$$

where $M_{1}$ is the diagonal lumped mass matrix. This scheme includes the consistent mass scheme $(\beta=1)$ and the lumped mass scheme $(\beta=0)$ as its special cases. We can obtain error estimates of the generalized mixed mass scheme, similar to those of the consistent mass scheme. 


\section{\$. Numerical Results}

In order to show the validity of our theory, numerical experiments are performed. We have used the FACOM 230-28 computer at Ehime University and the FACOM 230-75 computer at Kyushu University. Let $\Omega$ be a square domain defined by

$$
\Omega:-\pi / 2<x_{1}<\pi / 2, \quad-\pi / 2<x_{2}<\pi / 2 .
$$

Example.

$$
\begin{array}{ll}
\Delta \Delta u=\lambda u & \text { in } \Omega, \\
u=\partial u / \partial n=0 & \text { on } \partial \Omega .
\end{array}
$$

The above example is the same as the one given by Weinstein and Stenger ([13], p. 194). Although the exact eigenvalues for this problem are not known, Weinstein and Stenger have shown the lower and upper bounds for the exact ones by the method of intermediate problems and the Rayleigh-Ritz method, respectively.

The square domain is divided into uniform mesh with isosceles triangles as shown in Figure (36, 49, 64 and 81 nodes). The computations were performed for the parameter $\beta=0,0.5,1$. Table shows the results of the finite element solutions for the first four eigenvalues, compared with the lower and upper bounds for the exact values. It demonstrates that the approximate eigenvalues converge with the mesh size in good agreement with our theorem.

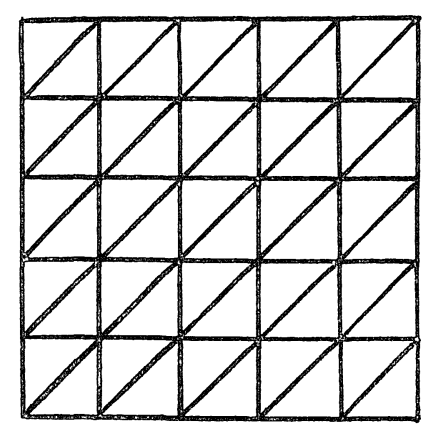

Figure. Mesh pattern (36 nodes) 
Table. The results of the eignvalues for Example

\begin{tabular}{|c|c|c|c|c|c|c|c|}
\hline \multirow{2}{*}{ mesh } & \multirow{2}{*}{ (node) } & \multirow{2}{*}{$h$} & \multirow{2}{*}{$\beta$} & \multicolumn{4}{|c|}{ eigenvalue (number) } \\
\hline & & & & 1 & 2 & 3 & 4 \\
\hline \multirow{3}{*}{\multicolumn{2}{|c|}{36}} & \multirow{3}{*}{$\frac{\sqrt{2 \pi}}{5}$} & 0 & 9. 7494 & 32.0009 & 32.0011 & 66.9656 \\
\hline & & & 0.5 & 12.5516 & 46.5794 & 51.2400 & 109.9170 \\
\hline & & & 1 & 17. 2642 & 77.6260 & 98.6773 & 222. 9727 \\
\hline \multirow{3}{*}{\multicolumn{2}{|c|}{49}} & \multirow{3}{*}{$\frac{\sqrt{2 \pi}}{6}$} & 0 & 10.5935 & 36.9346 & 36.9346 & 77. 6290 \\
\hline & & & 0.5 & 12.7965 & 49. 3474 & 53.0603 & 116. 5038 \\
\hline & & & 1 & 16. 0165 & 71.2238 & 84.5717 & 197. 3236 \\
\hline \multirow{3}{*}{\multicolumn{2}{|c|}{64}} & \multirow{3}{*}{$\frac{\sqrt{2} \pi}{7}$} & 0 & 11.1828 & 40.5867 & 40.5867 & 85.6151 \\
\hline & & & 0.5 & 12. 9371 & 50.9931 & 53. 9416 & 119. 2188 \\
\hline & & & 1 & 15. 2789 & 67.1105 & 76. 2634 & 178. 7896 \\
\hline \multirow{3}{*}{\multicolumn{2}{|c|}{81}} & \multirow{3}{*}{$\frac{\sqrt{2} \pi}{8}$} & 0 & 11.6053 & 43.3194 & 43.3195 & 91.6870 \\
\hline & & & 0.5 & 13.0252 & 52. 0410 & 54.4127 & 120.3749 \\
\hline & & & 1 & 14. 8062 & 64.3767 & 71.0464 & 165.8120 \\
\hline \multirow{2}{*}{\multicolumn{4}{|c|}{$\begin{array}{l}\text { lower bound for } \lambda_{i}^{*} \\
\text { upper bound for } \lambda_{i} *\end{array}$}} & 13. 2820 & 55.240 & 55.240 & 120.007 \\
\hline & & & & 13. 3842 & 56.561 & 56.561 & 124.074 \\
\hline
\end{tabular}

* From Weinstein and Stenger [13]

\section{Acknowledgements}

The author is grateful to Professor M. Yamaguti of Kyoto University and Professor T. Yamamoto of Ehime University for their continuous encouragements and helpful suggestions.

\section{References}

[1] Babuska, I. and Zlamal, M., Nonconforming elements in the finite element method with penalty, SIAM J. Numer. Anal., 10 (1973), 863-875.

[2] Ciarlet, P. G. and Raviart, P. A., A mixed finite element method for the biharmonic equation, Proceedings of the sy'mposium on mathematical aspects of finite elements in partial differential equations, (ed. Carl de Boor) Academic Press, 1974.

[3] Fix, G., Orders of convergence of the Rayleigh-Ritz and Weinstein-Bazley methods, Proc. Nat. Acad. Sci. U. S. A., 61 (1968), 1219-1223.

[4] Johnson, C., On the convergence of a mixed finite element method for plate bending problems, Numer. Math., 21 (1973), 43-62.

[5] Kikuchi, F., Notes on the lumped mass approximation for vibration problems, Theoretical and Applied Mechanics, 22, Univ. of Tokyo Press, (1974), 95-107.

[6] Kikuchi, F., Convergence of the ACM finite element scheme for plate bending problems, Publ. RIMS, Kyoto Univ., 11 (1975), 247-265.

[7] Kikuchi, F., On a finite element scheme based on the discrete Kirchhoff assumption, Numer. Math., 24 (1975), 211-231. 
[8] Mikhlin, S. G., Variational methods in mathematical physics, Pergamon Press, 1964.

[9] Miyoshi, T., A finite element method for the solutions of fourth order partial differential equations, Kumamoto J. Sci. (Math.), 9 (1972), 87-116.

[10] Miyoshi, T., A mixed finite element method for the solution of the von Kármán equations, Numer. Math., 26 (1976), 255-269.

[11] Piece, J. G. and Varga, R. S., Higher order convergence results for the Rayleigh-Ritz method applied to eigenvalue problems. I : estimates relating Rayleigh-Ritz and Galerkin approximations to eigenfunctions, SI AM J. Numer. Anal., 9 (1972), 137-151.

[12] Strang, G. and Fix, G., An analysis of the finite element method, Prentice-Hall, 1973.

[13] Weinstein, A. and Stenger, W., Methods of intermediate problems for eigenvalues, theory and ramifications, Academic Press, 1972.

[14] Wilkinson, J. H., The algebraic eigenvalue problem, Oxford Univ. Press, 1965.

[15] Ishihara, K., Convergence of the finite element method applied to the eigenvalue problem $\Delta u+\lambda u=0$, Publ. RIMS, Kyoto Univ., 13 (1977), 47-60. 